\title{
Case report: Ovarian cortex and stroma on an endometrial sampling
}

\author{
Suzanne Cao', Tina Paul ${ }^{2}$ and Karen Noblett ${ }^{3}$ \\ ${ }^{1}$ Resident Physician, Riverside University Health System - Medical Center, University of California, Riverside, CA, USA \\ ${ }^{2}$ Attending Physician, Riverside University Health System - Medical Center, University of California, Riverside, CA, USA \\ ${ }^{3}$ Department Chair, Riverside University Health System - Medical Center, University of California, Riverside, CA, USA
}

\begin{abstract}
Background: Ectopic ovarian tissue found within an endometrial sample has not be previously reported. The short and long term implications as well as management of this finding is unknown.

Case: We present a 30 year old, nulligravid, with a known history of leiomyomas, who underwent a hysteroscopy, dilation and curettage, and a myomectomy for an endocavitary leiomyoma. Microscopic pathology results of the endometrial sample demonstrated ovarian cortex and stroma.

Conclusion: The cause of this ectopic ovarian tissue could not be determined. She does not appear to have suffered any consequence of from this finding so far. However, any long term implications remain unknown. Until more information is known, no definitive management besides observation should be utilized and current guidelines should remain unchanged.
\end{abstract}

\section{Introduction}

Diagnostic and operative hysteroscopies (HSC) are common gynecologic surgeries. Common indications for this procedure include abnormal uterine bleeding, post-menopausal bleeding, endometrial polyps, submucosal fibroids, retained foreign bodies, and desire for sterilization. When an endometrial tissue sample is required for diagnostic purposes, a dilation and curettage (D\&C) is commonly performed. Complications of this procedure include uterine perforation, cervical laceration, infection and bleeding. Often, findings from a HSC plus D\&C will guide future treatment planning. In this case report, we present a patient who underwent a HSC D\&C for abnormal uterine bleeding with the findings of ovarian cortical and stromal tissue on curettage samples of the endometrium. To our knowledge, there has been not been a documented case report of ovarian tissue discovered on an endometrial sampling. The short and long term implications of this finding are unknown, as well as a lack of literature to inform or guide future management.

\section{Case}

We present a 30 year old African American, nulligravid female, who was initially seen in our Women's Health Clinic (WHC) as an Emergency Department (ED) follow-up. She had been seen in the ED two weeks prior, complaining of heavy vaginal bleeding associated with fatigue and dizziness. She was found to be anemic with a hemoglobin of $8.2 \mathrm{~g} / \mathrm{dL}$ and a hematocrit of $26.3 \%$ and was transfused two units of packed red blood cells for symptomatic anemia. She was discharged from the ED and advised to follow up in the WHC. Upon our initial encounter, she endorsed a history of heavy vaginal bleeding for the past six months. The flow was described as intermittent throughout the day and daily for the past six months and that she had been passing blood clots requiring several pads a day. She endorsed a history of fibroids resulting in a blood transfusion and a vaginal myomectomy five years prior. Her vaginal bleeding had improved after the myomectomy, but had progressively worsened over the past six months. She attempted oral contraceptives to help control the bleeding, but stated that they only made her symptoms worse and stopped the OCP's two months prior. The patient's past medical history and social history were noncontributory, and her only medication was an iron supplement.

Her abnormal uterine bleeding was initially worked up by her primary care provider and included a pelvic ultrasound and magnetic resonance imaging (MRI) two months prior to being seen in our clinic. Pelvic ultrasound demonstrated a $9.9 \times 8.4 \times 5.5 \mathrm{~cm}$ uterus with a hyper-vascular, hypoechoic structure seen within the endometrial cavity measuring $3.9 \times 5.6 \mathrm{~cm}$. The right ovary was $4.5 \times 3.3 \times 1.6$ $\mathrm{cm}$ and the left ovary was $3.7 \times 2.5 \times 2.6 \mathrm{~cm}$, both of which appeared normal (Figure 1). The MRI demonstrated a large heterogeneously enhancing endocavitary mass measuring $6 \times 4 \mathrm{~cm}$, compatible with a submucosal fibroid (Figure 2).

At the time of her initial clinic visit, options were discussed for the treatment of her abnormal uterine bleeding including medical management versus surgery. Patient was in a steady, long term relationship and desired future fertility within the next three years. She opted for surgical management to control her abnormal vaginal bleeding and to give her the best chance at future fertility.

The decision was made to take her to the operating room for a

Correspondence to: Suzanne Cao, Department of Obstetrics and Gynecology, Riverside University Health System - Medical Center, 26520 Cactus Ave., Moreno Valley, CA 92555, Tel: 415-992-2780; E-mail: suzcao@gmail.com

Key words: endometrium, hysteroscopy, ovary

Received: November 01, 2016; Accepted: November 14, 2016; Published: November 17, 2016 


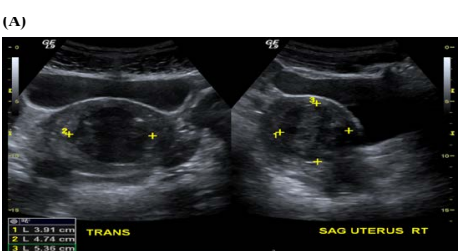

(B)
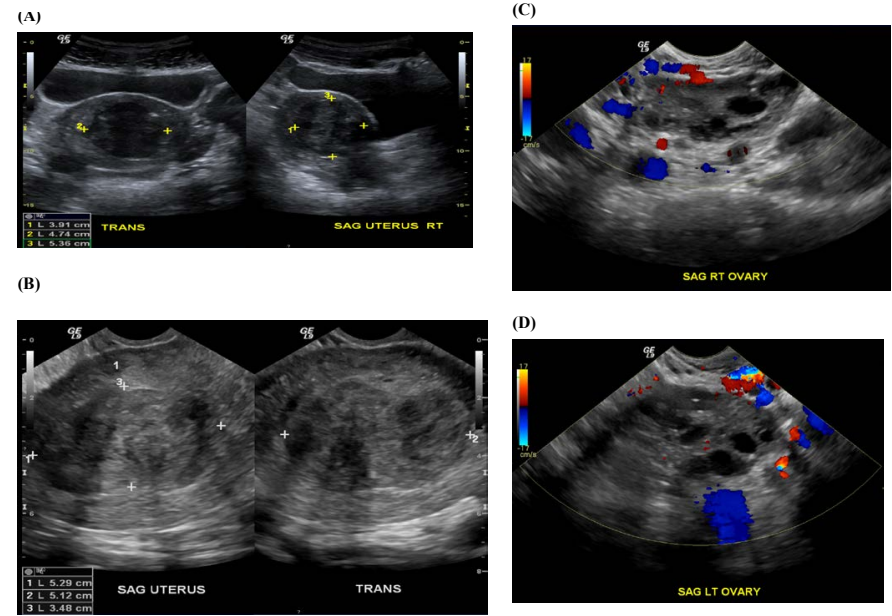

(D)

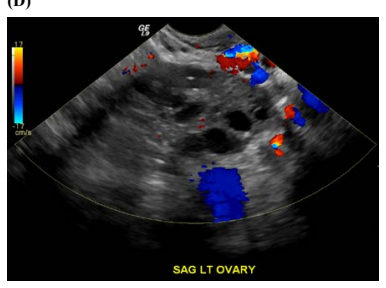

Figure 1. Ultrasound imaging showing transabdominal and transvaginal view of the endocavitary mass. A: transabdominal of the uterus, B: transvaginal of the uterus, C: transvaginal of the right ovary, D: transvaginal of the left ovary.

(A)
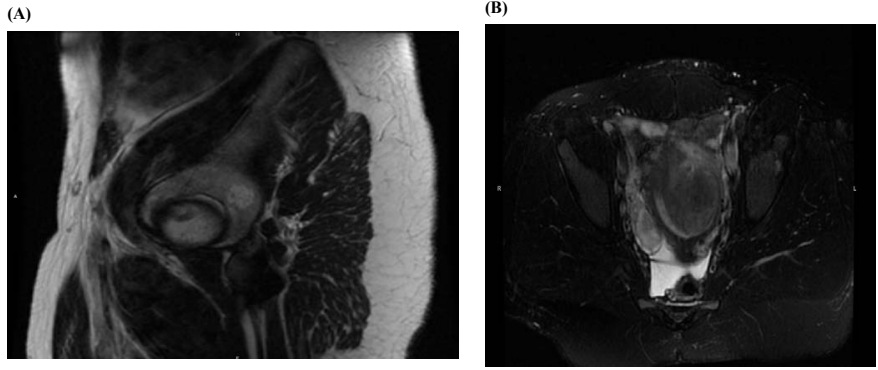

Figure 2. MRI images showing endocavitary mass. A: sagittal view, B: transverse view.
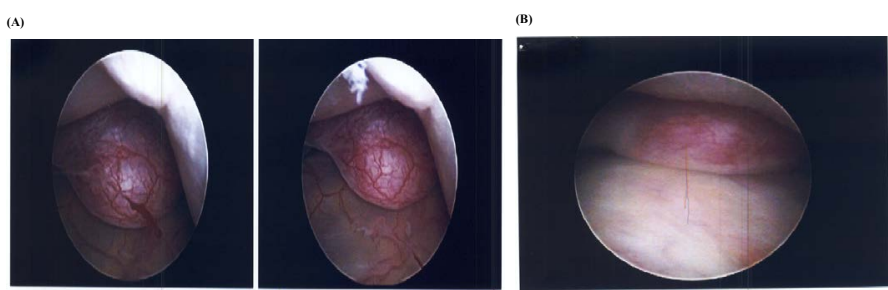

Figure 3. Images from the first hysteroscopy demonstrating a vascular polyp appearing mass at the fundus.

HSC, D\&C, and myomectomy. Upon entry into the uterine cavity, no definitive fibroid was visualized. However, a soft, vascular, polypoidappearing structure was visualized at the fundus (Figure 3). The mass was removed using a Myosure and a sharp D\&C was subsequently preformed without difficulty or intra-operative complications. The estimated blood loss was $50 \mathrm{~mL}$ and operative time was less than an hour. The patient was discharged home from the PACU.

The microscopic pathologic diagnosis demonstrated fragments of submucosal leiomyoma as well as fragments of ovarian tissue, including cortical and stromal tissue with no evidence of malignancy (Figure 4). Given the unusual pathology findings, the samples were sent for re-evaluation to an outside pathologist who confirmed the presence of ovarian tissue in the specimen. The patient was contacted and the pathology report was reviewed and she was given instruction on close follow-up.

At her follow up appointment, medical management was discussed to control her abnormal uterine bleeding until pregnancy was desired.
After discussion with her significant other, she reported they now desired to pursue pregnancy. To assess her anatomy and any evidence of perforation, we offered another HSC with concomitant laparoscopy (LSC) to give us direct visualization of the uterus during the HSC, and chromopertubation to assess tubal patency.

She was taken back to the operating room a week later. A concomitant HSC and LSC were performed. The uterus was successfully distended and upon entry into the peritoneum and uterus no obvious uterine perforation was noted. The polyp-appearing structure visualized on the prior HSC was not seen. A large hard mass consistent with a leiomyoma was now visualized in the cavity of the uterus along the anterior left lateral wall close to the fundus (Figure 5). This large mass was removed using a Myosure device under direct visualization with laparoscopy.

No evidence of intra-operative uterine perforation was noted. Bilateral ovaries were visualized, both of which appeared normal. Chromopertubation was performed with patency demonstrated bilaterally. Microscopic diagnosis on pathology of the second curettage was consistent with a leiomyoma, without any ovarian tissue seen. Findings were discussed with the patient, who was counseled to proceed with pregnancy as desired.

\section{Discussion}

We present a case of a 30 year old female who underwent a HSC and $\mathrm{D} \& \mathrm{C}$ for abnormal uterine bleeding, likely from a uterine leiomyoma

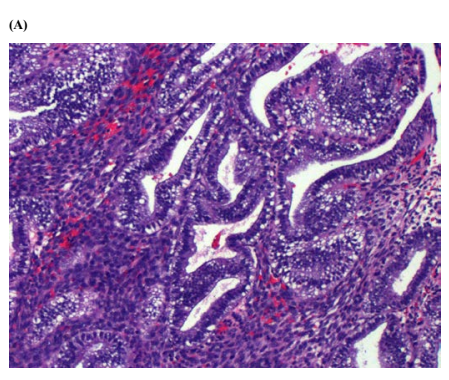
(B)
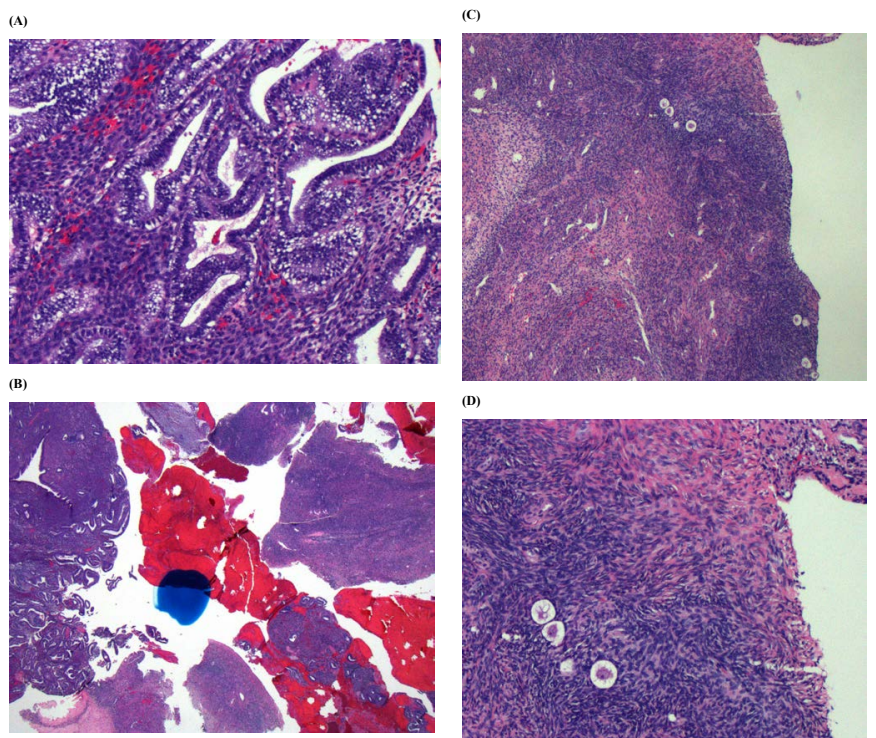

(D)

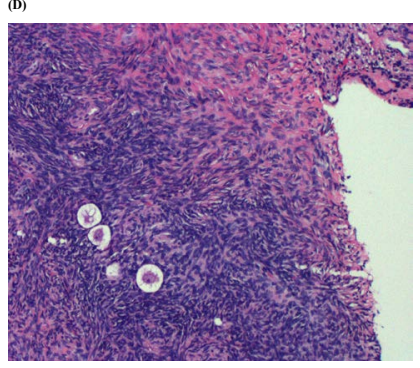

(E)

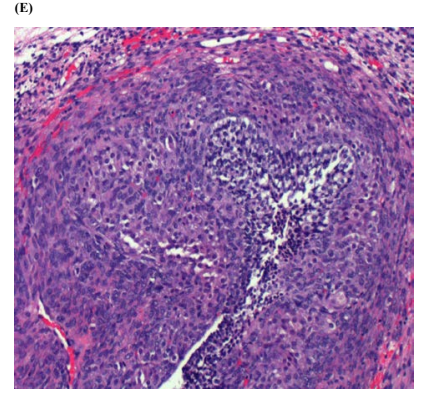

Figure 4. Microscopic images of the endometrial sample. A: secretory endometrium (100x), B: endometrium (right) and ovary (left) $(12.5 \mathrm{x}), \mathrm{C}$ : ovarian cortex with primordial follicles (40x), D: ovarian cortex with primordial follicles (100x), E: developing follicle with theca luteinization $(100 x)$. 
(A)

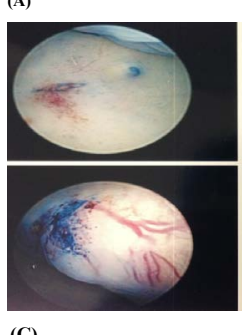

(C)

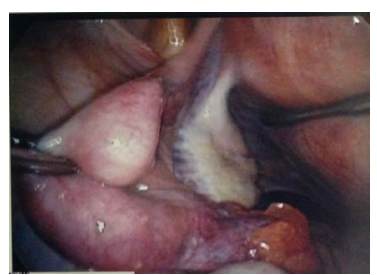

Figure 5. Images from the second hysteroscopy and laparoscopy. A: fibroid on the left lateral wall, B: patent bilateral fallopian tubes and normal appearing ovaries.

seen on imaging. The pathology report demonstrated ovarian cortical and stromal tissue on the endometrial sample. To our knowledge, there have been no reported cases of this occurrence. The etiology of ovarian tissue in the endometrium and the long-term implications of its ectopic location are unknown. Management of this finding as well as its effect on OBGYN problems is also unknown.

A possible theory to explain this finding of ovarian tissue on our endometrial sample is uterine perforation which is one of the most common complications of HSC. Many perforations are asymptomatic and therefore underreported and thus remain undetected. If detected, many cases of perforation are managed conservatively as most perforations are uncomplicated and do not require additional intervention. Most studies demonstrate an incidence of approximately $1 \%$ for all operative HSC [1]. Although the rate of this complication is low, it can be potentially life threatening. Perforation is usually recognized when an instrument passes beyond the depth of the uterine fundus, there is loss of visualization, internal structures are seen at the fundus, or when there is a sudden increase in the fluid deficit. The most common site of perforation is the uterine fundus, but there is typically minimal bleeding. The potential for serious injury is increased if electrosurgical energy, morcellation, or if sharp or suction curettage is utilized during the procedure. In this case, we did not suspect uterine perforation during the initial HSC D\&C and saw no evidence of uterine perforation during the subsequent HSC or LSC, which was performed just one week following the initial surgery. There is one case report of uterine perforation resulting in ovarian migration to the site of perforation with delayed presentation. Su et al. [2] described a case of a patient who had undergone a D\&C for an adherent placenta and retained products of conception. Approximately two years later, she presented with a hyper-echogenic mass in the uterus diagnosed by ultrasound. Ultrasound findings were consistent with an abnormal uterine mass containing several follicles emanating from the right posterior wall of the uterine cavity and absence of myometrial tissue. A HSC and LSC demonstrated uterine perforation with ovarian incarceration. The patient had remained asymptomatic throughout the entire two years. Our suspicion that perforation caused this pathology finding is extremely low given the negative findings on both repeat HSC and LSC. We were unable to find any case reports of ovarian tissue on an endometrial sample with or without uterine perforation evident. Most uterine perforations result in adipose or bowel tissue being present in an endometrial sample. Any perforation with damage to internal structures would necessitate the need for hospital admission. The consequences of internal organ injury can be life threatening and exploratory laparotomy should be immediately considered if the patient develops any signs of injury.

Another theory to explain the presence of ovarian tissue in our curettage is the presence of ectopic ovarian tissue. Kuwamura et al. [3] describes the occurrence of ectopic ovarian tissue in cynomolgus monkeys. With an incidence rate of $7.6 \%$, this ectopic tissue was an incidental finding in the parametrium by microscopic examination. It is believed that this ectopic ovarian tissue may result from disturbed migration of the ovary during embryogenesis. The mesothelium just medial to the mesonephros, is a transitory structure important in the development of the kidney, and will thicken to develop into paired gonadal ridges, the medial portion of which is the origin of the gonads. The gonads will descend from the posterior abdominal wall into the pelvis into the ovarian fossae. Uterine development occurs from fusion of the paramesonephric/mullerian ducts that arise from the mesoderm lateral the mesonephric ducts and mesonephros. This article cites the presence of ovarian tissue outside of the endometrium likely through accidental implantation during embryogenesis. We did not visualize ovarian tissue on either during both HSC and both ovaries could were be clearly visualized on pelvic ultrasound clearly. We can hypothesize the possibility of abnormal embryonal gonadal migration or the incorporation of gonadal tissue as the paramesonephric ducts move medially to fuse resulting in the presence of ovarian tissue within the endometrium.

Regardless of how ovarian tissue ended up in our endometrial sampling, the patient did not appear to suffer any ill effects from this. She had normal sexual development, hormonal drive, and normal menses prior to her diagnosis of leiomyomas. The long-term implications of this finding are unknown. Until more information becomes available, no definitive management other than observation should be utilized and current guidelines for OBGYN problems should remain unchanged. Given the normal pathology on the second sample, we do not anticipate any effects on her fertility. The patient ultimately completed surgical management of her submucosal leiomyoma, suffered no adverse effects from either surgery and demonstrated marked improvement of her abnormal uterine bleeding. No further treatment or contraception was offered since the patient desired to get pregnant in the immediate future. We plan to continue to follow her closely.

\section{References}

1. Jansen FW, Vredevoogd CB, van Ulzen K, Hermans J, Trimbos JB, et al. (2000) Complications of hysteroscopy: a prospective, multicenter study. Obstet Gynecol 96: 266. [Crossref]

2. Su S, Tao G, Dong B, Shi L, Dong J (2015) Case Report: Delayed presentation of uterine perforation with ovary migration after dilation and curettage. Int J Clin Exp Med 8: 6311.

3. Kuwamura Y, Kakehi K, Hirakawa K, Miyajima H (2006) Ectopic Uterine Ovarian Tissue in Cynomolgus Monkeys. Toxicol Pathol 34: 220. [Crossref]

Copyright: (C2016 Cao S. This is an open-access article distributed under the terms of the Creative Commons Attribution License, which permits unrestricted use, distribution, and reproduction in any medium, provided the original author and source are credited. 